\title{
Análisis comparativo de inhibidores verdes vs inhibidores tradicionales en la corrosión de los metales
}

\section{Comparative analysis of green inhibitors vs. inhibitors traditional in metal corrosion}

\author{
Zariyen Agraje ${ }^{1}$ Ismael Barahona ${ }^{l}$, Joseph Quíroz ${ }^{1}$, Héctor Pino ${ }^{1}$, Cecilio Hernández $z^{{ }^{*}}$, Casilda Saavedra ${ }^{*}$ \\ ${ }^{1}$ Licenciatura en Ingeniería Civil - Facultad de Ingeniería Civil-Centro Experimental de Ingeniería \\ Universidad Tecnológica de Panamá
}

\begin{abstract}
Resumen Los inhibidores industriales más utilizados para disminuir la corrosión son aquellos que contienen heteroátomos (tales como N, S, P, O), provocando daños al ambiente, por lo cual se ha optado por alternativas naturales. Los inhibidores verdes son una alternativa amigable para el medio ambiente, ya que nos permiten disminuir ciertos problemas que están afectando nuestro planeta, mediante el uso excesivo de los productos químicos. Esta investigación consiste en la comparación de inhibidores verdes a base de frutas (aguacate y manzana verde) versus los inhibidores tradicionales. El diseño de este estudio es experimental, con tres tratamientos y un control. Los tratamientos consistieron de tres tipos de inhibidores verdes y un inhibidor de uso industrial. Los inhibidores verdes fueron preparados respectivamente, con los siguientes materiales naturales: pulpa de aguacate, extracto de la cascara de manzana verde y pulpa de la manzana verde. Con estos 3 tipos de inhibidores verdes se pudo estudiar los niveles de corrosión en los metales, mediante la pérdida de peso presentada en las láminas de acero al carbono A36. Estas placas metálicas fueron expuestas a un ambiente marino y a un ambiente controlado en el laboratorio. Al finalizar nuestra investigación pudimos apreciar que el inhibidor verde a base de la pulpa de aguacate fue el más efectivo, ya que pudo contrarrestar con mayor eficiencia la velocidad de corrosión en las placas metálicas. Si bien, no tienen un porcentaje de efectividad como el inhibidor industrial, pero si puede ser utilizado como alternativa para mitigar el proceso de corrosión.
\end{abstract}

Palabras clave Acero al carbono, corrosión, inhibidor, salinidad.

\begin{abstract}
The most widely used industrial inhibitors to decrease corrosion are those that contain heteroatoms (such as N, S, P, O), causing damage to the environment, which is why natural alternatives have been chosen. Green inhibitors are a friendly alternative for the environment, since they allow us to reduce certain problems that are affecting our planet, through the excessive use of chemical products. This research involves comparing green fruit-based inhibitors versus traditional inhibitors. The design of this study is experimental, with three treatments and one control. Treatments consisted of three types of green inhibitors and one inhibitor for industrial use. The green inhibitors were prepared respectively with the following natural materials: avocado pulp, extract of the green apple peel and pulp of the green apple. With these 3 types of green inhibitors, it was possible to study the levels of corrosion in metals, through the weight loss presented in A36 carbon steel sheets. These metal plates were exposed to a marine environment and to a controlled laboratory environment. At the end of our investigation, we could see that the green inhibitor based on the avocado pulp was the most effective, since it could more efficiently counteract the corrosion rate in the metal plates. Although, they do not have a percentage of effectiveness as the industrial inhibitor, but it can be used as an alternative to mitigate the corrosion process.
\end{abstract}

Keywords Carbon Steel, corrosion, inhibitor, salinity.

* Corresponding author: correo_electrónico_casilda.saavedra@utp.ac.pa

\section{Introducción}

La corrosión en los metales es un proceso natural e inevitable causado por varios agentes como: lluvia, temperatura, humedad, salinidad, ácidos y la contaminación.
Panamá, siendo un país tropical, cálido y sometido a la constante influencia del mar, constituye un medio altamente agresivo para la corrosión de los materiales metálicos, presentes, sobre todo, en aquellas grandes obras que contribuyen al desarrollo de la economía como: los puentes 
sobre el canal, las estructuras metálicas presentes en edificaciones, que cada vez son más numerosas [1].

La corrosión en los metales siempre ha estado presente a nivel mundial, por lo que se han realizo múltiples estudios, con el fin de contrarrestar este proceso a través de inhibidores y de esta manera proporcionarle más años de utilidad a los mismos evitando que el acero pierda su resistencia.

Las nuevas tendencias en inhibidores de la corrosión son el uso de aminoácidos, siendo los compuestos orgánicos más eficientes aquellos que contienen heterotermos (como N, S, P, $\mathrm{O})$. Se reporta que en los compuestos orgánicos que tienen $\mathrm{OH},-\mathrm{COOH}, \mathrm{NH} 2$, son excelentes inhibidores de la corrosión, especialmente en medios ácidos [2]. Lo que preocupa es que la gran mayoría de estos compuestos son contaminantes y afectan directamente el medio ambiente y los seres vivos.

Conociendo el alto índice de contaminación que producen estos anticorrosivos industriales, se ha optado por elaborar inhibidores más adecuados y económicos que ayuden a prevenir la temprana corrosión en los metales y que sean amigables con el medio ambiente.

Estos inhibidores conocidos como "inhibidores verdes" no le causan daños al ambiente y actualmente existen una cantidad de ellos procedentes de plantas y frutas. En el 2007, Cecilio Hernández [1] utilizó cinco plantas naturales (tuna, pitahaya, herrero, acacia y cedro), llegando a la conclusión de que, en un ambiente poco agresivo (estación de Panamá), donde hay ciclos de humedad y secado; al año de exposición, el cedro es el más efectivo. Por otro lado, en ambiente agresivo (estación de Colón), también el cedro muestra mayor efectividad, pero en un menor rango que en Panamá.

En el 2013 [3], al terminar su proceso de investigación concluyen que: "El jugo de albaricoque actúa como un inhibidor de la corrosión del acero suave con una eficacia de inhibición máxima al $75 \%$ a $30^{\circ} \mathrm{C}$ y el nivel máximo de concentración del inhibidor".

En estudios realizados en el 2016 [4], se indica que la cáscara de la nuez de la India (Anacardium occidentale), es efectiva para el tratamiento superficial de metales, ya que su aceite obtenido, protege los metales de la corrosión. De igual forma, en el 2016, Virginia Gómez [5], utilizó cáscaras de manzana como inhibidor verde ante un medio corrosivo formado por una solución de ácido clorhídrico $(\mathrm{HCl}) 0.5 \mathrm{M} \mathrm{a}$ temperatura ambiente. Mediante los resultados obtenidos por medio de espectroscopía de impedancia electrónica, curvas de polarización potenciodinámicas y pérdida de peso, muestran tendencias similares conforme aumenta la concentración del extracto. Durante las pruebas, la máxima eficiencia es por pérdida de peso, obtenida a cuatro horas, $25^{\circ} \mathrm{C}$ con $500 \mu \mathrm{L}$ de extracto. Para esto, utilizaron diferentes solventes, entre ellos el metanol y la acetona; concluyendo que la acetona era más efectiva en medios orgánicos y el metanol en medios ácidos.
En otros estudios realizados en el 2013[6], se encontró que a partir del estudio fitoquímico el extracto de aguacate posee mayor concentración de taninos que el extracto de tabaco. Por el estudio de pérdida de peso, se observó que el aguacate actúa con mayor eficiencia en el acero al carbono que en el aluminio, se obtuvo un porcentaje de inhibición de hasta el 98.1\%. Esto se debe a una mayor reactividad del acero al carbono con los taninos del extracto de aguacate.

Conociendo el tipo de clima presente en Panamá, se decidió enfocar la investigación en la problemática que afecta el acero al carbono A36; metal utilizado a diario en obras civiles, donde se encuentra expuesto directamente a la corrosión atmosférica. Se busca determinar la efectividad de los inhibidores verdes de aguacate y manzana verde comparados a los inhibidores tradicionales.

La hipótesis de investigación, $\mathrm{H}_{1}$, es que los inhibidores verdes a base de extractos de manzana verde y aguacate son tan efectivos como los inhibidores industriales de la corrosión. La hipótesis nula, $\mathrm{H}_{0}$, es que los inhibidores verdes no son tan efectivos como los inhibidores industriales de la corrosión.

\section{Materiales y metodología}

El diseño de esta investigación es experimental y cuantitativa. Es una investigación de transición.

La ejecución de este se llevó a cabo en tres etapas: preparación de las muestras, exposición de las láminas y recolección de datos.

2.1 Materiales
Láminas de acero al carbono
Jabón ALCONOX
Agua desionizada
Etanol a 96\%
Solución de HCL
$\mathrm{Sb}_{2} \mathrm{O}_{3}$
$\mathrm{SnCl}_{2}$
Anticorrosivo industrial (Tipo Minio)
Persea americana (Aguacate)
- Vranny Smith (Manzanas verdes)
- Balanza digital
15 libras de sal (NaCl)
Cámara de salinidad

\subsection{Metodología}

Se utilizaron un control y dos tratamientos.

El control consistió en una lámina de acero A36 sometida a ambiente corrosivo sin inhibidores.

Los tratamientos fueron los siguientes: 1) láminas de acero A36 sometidas a inhibidor de la corrosión consistente de extracto y cáscara de manzana verde y 2) láminas A36 con aplicación de inhibidor consistente de extracto de aguacate. 
Las modalidades de investigación son: un ambiente controlado en el laboratorio del Centro Experimental de Ingeniería de la Universidad Tecnológica de Panamá y un ambiente costero natural, en la estación experimental de la UTP en Sherman, Colón (figura 1).

La estación experimental rompeolas Sherman se encuentra localizada con una latitud y longitud de $9^{\circ} 22^{\prime} 21^{\prime \prime}$ Norte, $79^{\circ}$ 6' 48" Oeste, respectivamente. La acción de los vientos mantiene las olas chocando contra las rocas que sirven de rompeolas lo cual produce abundante sal en forma de rocío y una atmósfera con un alto contenido humedad. La humedad relativa sobrepasa el $80 \%$, la mayor parte del año [7].

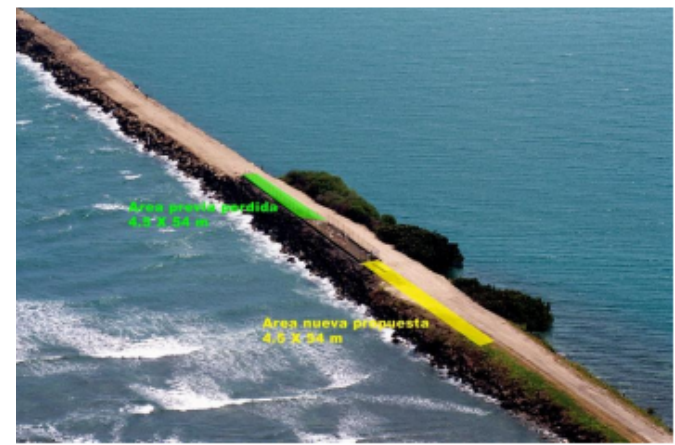

Figura 1. Estación rompeolas en Sherman (vista aérea) [7].

Por otro lado, el ambiente controlado fue la cámara de salinidad (figura 2) ubicada en los laboratorios, del Centro Experimental de Ingeniería, de la Universidad Tecnológica de Panamá extensión de Tocumen.

Esta cámara evaluó el comportamiento del material en un ambiente químicamente activo, bajo la exposición al Cloruro de sodio $(\mathrm{NaCl})$. Con ella se pueden acelerar los efectos del ataque por corrosión, teniendo en cuenta que una duración de 10 horas en la cámara de salinidad, equivale a un año de exposición al ambiente marino [8].

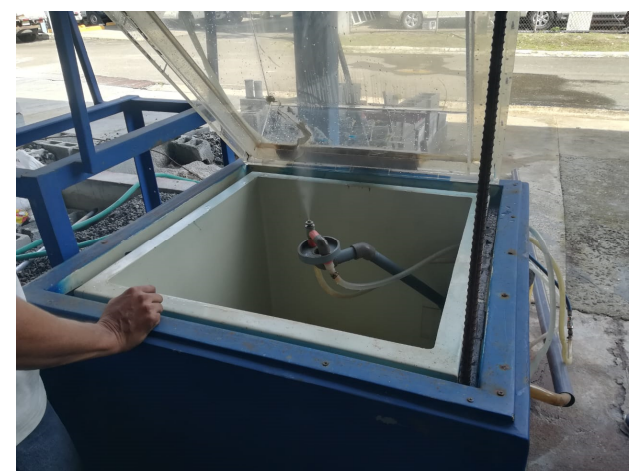

Figura 2. Cámara de salinidad utilizada.

\subsubsection{Preparación de las muestras}

1. Se cortaron 21 láminas de acero al carbono A36 aproximadamente a $100 \mathrm{mmx} 150 \mathrm{~mm}$, con espesor de $1 / 16$.
2. Se lavaron las 21 láminas de acero al carbono con jabón ALCONOX para eliminar la capa de grasa.

3. Se eliminó la capa de fosfato, la cual evita que se corroa con el procedimiento de decapado, preparando la solución mediante la norma ASTM G-1 [9], (figura 3).
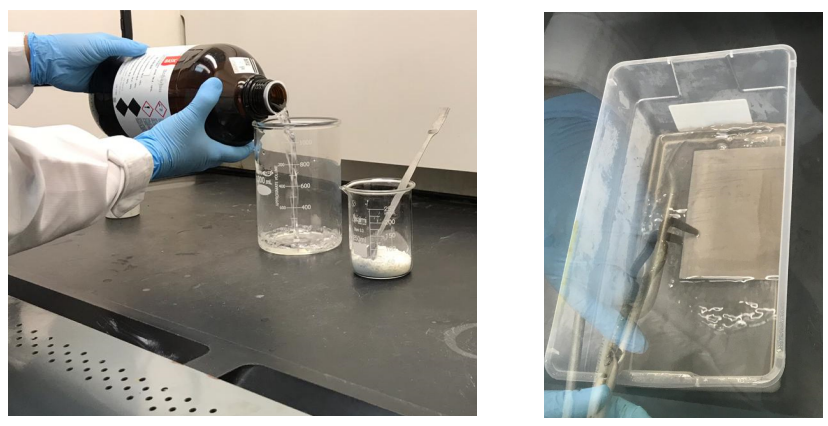

Figura 3. Preparación de la solución para realizar el decapado de las láminas.

4. Luego se limpiaron las láminas con etanol a 96\%, para eliminar restos de la solución.

5. Se pesaron las 21 láminas y después las dimensionamos para saber su verdadero ancho y longitud.

6. Se procedió a preparar los inhibidores verdes en los laboratorios de Análisis Industriales y Ciencias Aplicadas (LABAICA) ubicado en el Centro Experimental de Ingeniería de la Universidad Tecnológica, de la siguiente manera:

a. Solución de Persea americana (Aguacate).

- Se pesaron $250 \mathrm{~g}$ de pulpa, a la cual se le adicionaron $150 \mathrm{ml}$ de agua desionizada.

- Se homogenizo la solución en una licuadora industrial hasta obtener una solución pastosa.

b. Solución de Granny Smith (Manzana Verde).

- Pulpa

- $\quad$ Se pesaron $250 \mathrm{~g}$ de la pulpa, en la que añadimos $150 \mathrm{ml}$ de agua desionizada.

- Se unifico en una licuadora industrial hasta conseguir una consistencia pastosa parecida a la de las pinturas.

c. Cáscara

- Se pesaron $250 \mathrm{~g}$ de la cascara, a la que se agregaron $150 \mathrm{ml}$ de agua desionizada.

- Se llevó a ebullición esta mezcla para obtener los compuestos químicos de la cáscara de forma concentrada.

7. Se aplicaron aproximadamente tres $\mathrm{ml} \mathrm{de}$ anticorrosivo (figura 4) a las láminas de acero A36 por cara de la siguiente manera:

a. Cuatro láminas con anticorrosivo industrial, marca Glidden (Tipo minio).

b. Cuatro láminas con extracto de Persea americana.

c. Cuatro láminas con extracto de Granny Smith (figura 5). 


\section{d. - Cuatro láminas con cáscara de Granny Smith. \\ e. - Se dejaron Cuadro láminas, control, sin inhibidores aplicados.}

8. Se dejaron secar las láminas por aproximadamente 19 horas antes de exponerlas al ambiente.

9. Se preparó una solución salina basada en la norma ASTM B117-16 [10], para obtener el porcentaje de sal, la cual se aplicaría en la cámara de salinidad.

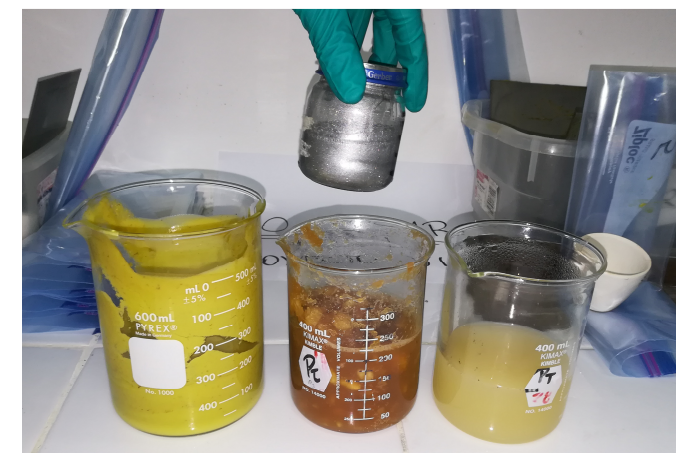

Figura 4. Inhibidores utilizados.

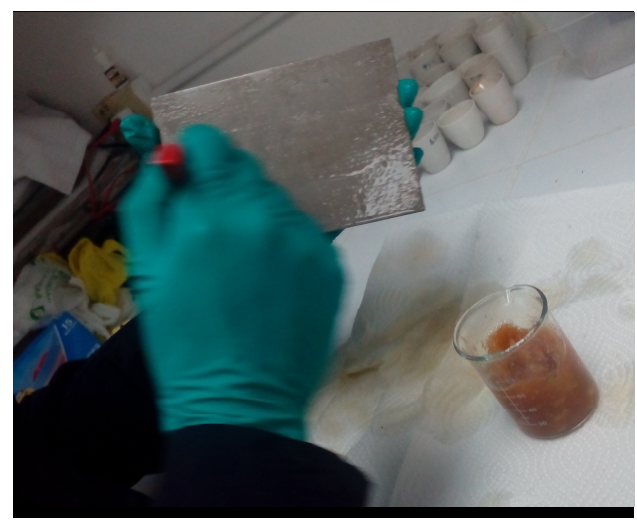

Figura 5. Aplicación de los inhibidores a las láminas.

\subsubsection{Exposición de las Láminas}

1. Se dejaron 10 láminas en la Cámara de salinidad ubicada en el centro experimental de Tocumen, en la que se colocaron dos láminas de cada una de las especificadas en el punto ocho (figura 6).

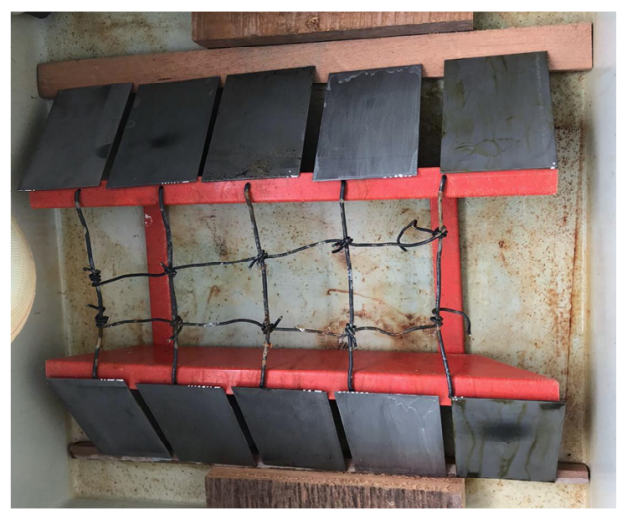

Figura 6. Colocación de las láminas en la cámara de salinidad.

2. Se llevaron 10 láminas a la estación del rompeolas, ubicado en los predios que tiene la Universidad Tecnológica de Panamá en Sherman, Colón. En la que colocamos dos láminas de cada una de las especificadas en el punto ocho (figura 7).

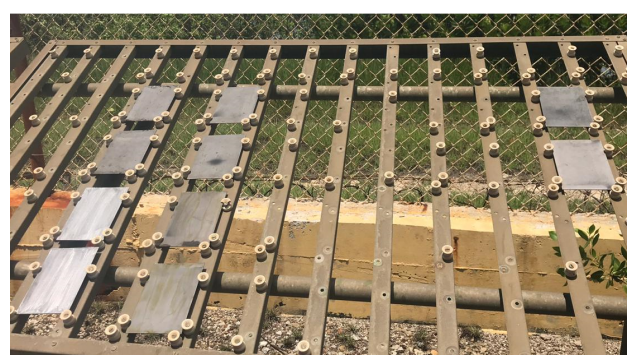

Figura 7. Colocación de las láminas en la estación rompeolas, Sherman.

\subsubsection{Recolección de datos}

1. Se retiraron las placas expuestas en ambos ambientes (figura 8).

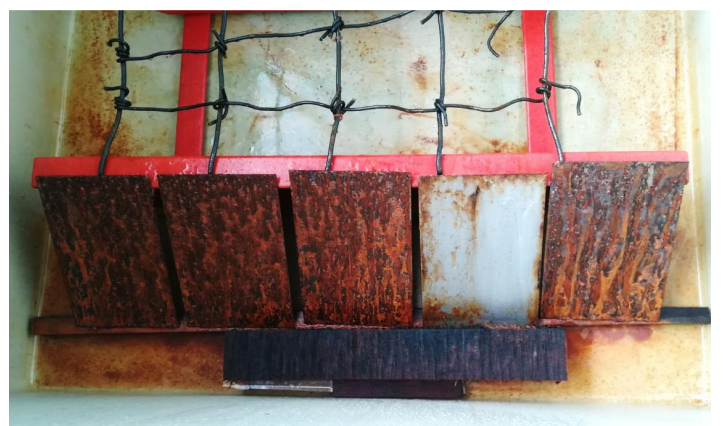

Figura 8. Láminas expuestas en la cámara de salinidad al finalizar el proyecto.

2. Se rasparon las láminas para quitar un poco de la corrosión.

3. Se realizó el decapado de las láminas de acero A36 para determinar la cantidad de peso que perdió la muestra por la corrosión (figura 9). 


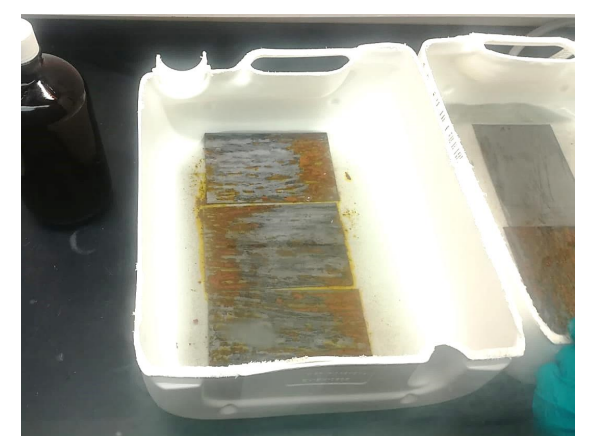

Figura 9. Decapado final de las láminas.

4. Se pesaron las láminas y se anotó su nuevo peso, para realizar el método gravimétrico descrito en la norma ASTM G-31[11].

En esta investigación nos referimos a la velocidad de corrosión, como el espesor perdido, en $\mu \mathrm{m} /$ horas. La velocidad se obtiene entonces a partir de la masa perdida (mg) mediante la relación

$$
\mathrm{V}=10 * \mathrm{~m} / \mathrm{A} * \rho * \mathrm{t}
$$

Donde:

$\mathrm{V}=$ velocidad expresada en $\mu \mathrm{m} / \mathrm{horas}$

$\mathrm{m}=$ masa perdida en $\mathrm{mg}$

$\mathrm{A}=$ Área de superficie total de la probeta en $\mathrm{cm}^{2}$

$\rho=$ densidad del metal en $\mathrm{g} / \mathrm{cm}^{3}$

$\mathrm{t}=$ tiempo de exposición en horas [12]

Con la ecuación (2) se calcula la eficiencia de inhibición del extracto.

$$
\% \mathrm{\eta}=\left[\left(\mathrm{V}_{\text {blanco })}-\left(\mathrm{V}_{\text {inh }}\right) / \mathrm{V}_{\text {blanco }}\right] * 100\right.
$$

Donde:

$\mathrm{V}_{\text {blanco }} \mathrm{y} \mathrm{V}_{\text {inh }}$, son las velocidades de corrosión sin inhibidor y con inhibidor, respectivamente [5].

\section{Resultados}

A continuación, los resultados basados en la exposición de las láminas de acero, expuestas a un ambiente costero natural y ambiente controlado (cámara de salinidad).

Las láminas estuvieron expuestas en dos periodos de 144 y 408 horas en la estación rompeolas de Sherman, y 12 y 24 horas en la cámara de salinidad. Donde luego de este tiempo se obtiene la velocidad de corrosión.

\subsection{Estación rompeolas en Sherman}

En estudios previos, se observó una especie de desgaste físico del recubrimiento conocido como erosión, posiblemente debido a las corrientes de viento que arrastran las partículas de sal [7]. La tabla 1 y la tabla .2 muestran los porcentajes de eficiencia de inhibición, de las láminas que estuvieron

\begin{tabular}{|c|c|c|c|c|c|}
\hline Inhibidor & Peso (g) & Peso final (g) & Área $(\mathrm{cm} 2)$ & $\begin{array}{l}\text { Velocidad de } \\
\text { corrosión } \\
(\mathrm{mg} / \mathrm{cm} \text { *h) }\end{array}$ & $\begin{array}{l}\text { \% Eficiencia } \\
\text { de inhibición }\end{array}$ \\
\hline Aguacate & 203.85 & 203.56 & 164.78 & $1.55492 \mathrm{E}-07$ & 41.4579439 \\
\hline Industrial & 204.07 & 204 & 167.4 & $3.69452 \mathrm{E}-08$ & 86.0903226 \\
\hline $\begin{array}{l}\text { Pulpa de } \\
\text { manzana }\end{array}$ & 203.47 & 203.07 & 163.24 & $2.16495 \mathrm{E}-07$ & 18.490566 \\
\hline $\begin{array}{l}\text { Cascara } \\
\text { Manzana }\end{array}$ & 204.14 & 203.75 & 164.78 & $2.0911 \mathrm{E}-07$ & 21.271028 \\
\hline Sin inhibidor & 204.58 & 204.08 & 166.32 & $2.65608 \mathrm{E}-07$ & 0 \\
\hline
\end{tabular}
expuestas en periodos de 144 y 408 horas.
Tabla 2. Exposición a 408 horas en la estación rompeolas

\begin{tabular}{|c|c|c|c|c|c|}
\hline Inhibidor & Peso (g) & Pesofinal $(\mathrm{g})$ & Área $(\mathrm{cm} 2)$ & $\begin{array}{c}\text { Velocidad de } \\
\text { corrosión } \\
(\mathrm{mg} / \mathrm{cm} * \mathrm{~h})\end{array}$ & $\begin{array}{c}\text { \% Eficiencia } \\
\text { de inhibición }\end{array}$ \\
\hline Aguacate & 197.02 & 196.37 & 161.7 & $1.25349 \mathrm{E}-07$ & 66.3766014 \\
\hline Industrial & 197.46 & 197.16 & 162.75 & $5.74801 \mathrm{E}-08$ & 84.5816276 \\
\hline $\begin{array}{c}\text { Pulpa de } \\
\text { manzana }\end{array}$ & 196 & 194.56 & 161.7 & $2.77696 \mathrm{E}-07$ & 25.51124 \\
\hline $\begin{array}{c}\text { Cascara } \\
\text { Manzana }\end{array}$ & 195.57 & 194.03 & 161.7 & $2.96981 \mathrm{E}-07$ & 20.3384095 \\
\hline Sin inhibidor & 204.43 & 202.46 & 164.78 & $3.72803 \mathrm{E}-07$ & 0 \\
\hline
\end{tabular}

De las tablas anteriores se puede observar que la velocidad de corrosión a mayor tiempo de exposición aumenta su velocidad. Además, observamos que, de los diferentes tipos de inhibidores verdes utilizados, el más eficiente, de los estudiados, es el aguacate el cual presento mayor porcentaje de eficiencia.

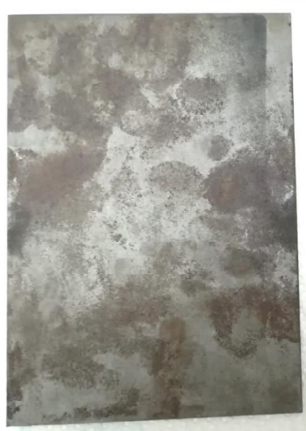

[a]

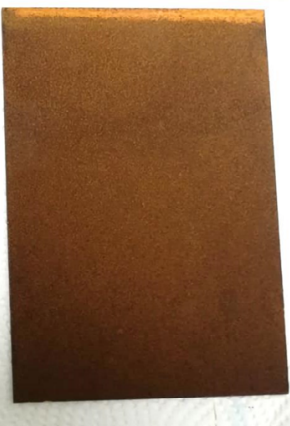

[b]
Figura 10. Comparación de placa sin exponer [a] y placa expuesta en la estación rompeolas, Sherman [b].

Para apreciar mejor los resultados de la pérdida de peso en las láminas de acero A36, se presentaron los pesos obtenidos al inicio y al final del proceso de decapado de las muestras (figura 11).

Tabla 1. Exposición a 144 horas en la estación rompeolas 


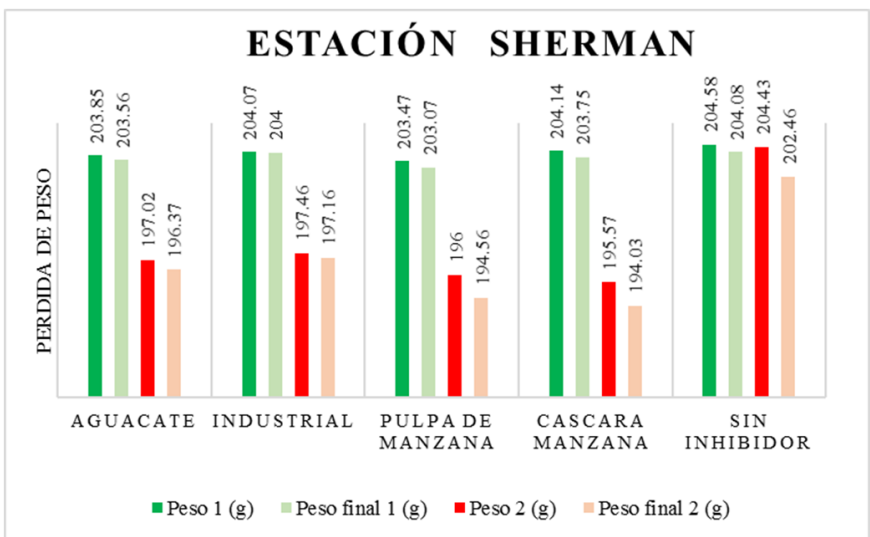

Figura 11. Pérdida de peso en láminas expuestas en ambiente costero natural.

\subsection{Ambiente controlado-cámara de salinidad}

La utilización de este ambiente controlado se usó para acelerar el proceso de corrosión. En estudios previos se muestra una pérdida de peso considerable en probetas expuestas a este medio agresivo [7].

Las tablas 3 y. 4 muestran los porcentajes de eficiencia de inhibición, de las láminas que estuvieron expuestas por un periodo entre 12 y 24 horas.

Tabla 3.-Exposición a 12 horas en la cámara de salinidad

\begin{tabular}{|c|c|c|c|c|c|}
\hline Inhibidor & Peso $(\mathrm{g})$ & Peso final $(\mathbf{g})$ & Área $(\mathbf{c m 2})$ & $\begin{array}{c}\text { Velocidad de } \\
\text { corrosión } \\
\left(\mathbf{m g} / \mathrm{cm}^{*} \mathbf{h}\right)\end{array}$ & $\begin{array}{c}\text { \% Eficiencia } \\
\text { de inhibición }\end{array}$ \\
\hline Aguacate & 198.03 & 195.61 & 163.77 & $1.56667 \mathrm{E}-05$ & 52.2117604 \\
\hline Industrial & 195.3 & 194.94 & 161.7 & $2.36042 \mathrm{E}-06$ & 92.8 \\
\hline $\begin{array}{c}\text { Pulpa de } \\
\text { manzana } \\
\text { Cascara }\end{array}$ & 204.46 & 199.54 & 166.32 & $3.13629 \mathrm{E}-05$ & 4.33333333 \\
\hline Manzana & 202.57 & 197.56 & 163.71 & $3.24458 \mathrm{E}-05$ & 1.03023639 \\
\hline Sin inhibidor & 196.61 & 191.61 & 161.7 & $3.27836 \mathrm{E}-05$ & 0 \\
\hline
\end{tabular}

Tabla 4. - Exposición a 24 horas en la cámara de salinidad

\begin{tabular}{|c|c|c|c|c|c|}
\hline Inhibidor & Peso $(\mathbf{g})$ & Pesofinal $(\mathbf{g})$ & Área $(\mathrm{cm} 2)$ & $\begin{array}{c}\text { Velocidad de } \\
\text { corrosión } \\
\left(\mathbf{m g} / \mathrm{cm}^{*} \mathbf{h}\right)\end{array}$ & $\begin{array}{c}\text { \% Eficiencia } \\
\text { de inhibión }\end{array}$ \\
\hline Aguacate & 204.47 & 200.42 & 167.4 & $1.28252 \mathrm{E}-05$ & 29.0000585 \\
\hline Industrial & 204.41 & 204.11 & 165.85 & $9.58897 \mathrm{E}-07$ & 94.6915932 \\
\hline $\begin{array}{c}\text { Pulpa de } \\
\text { manzana } \\
\text { Cascara }\end{array}$ & 197.64 & 192.88 & 162.75 & $1.55043 \mathrm{E}-05$ & 14.1689597 \\
\hline Manzana & 199.37 & 193.99 & 165.85 & $1.71962 \mathrm{E}-05$ & 4.80257071 \\
\hline Sin inhibidor & 197.37 & 191.86 & 161.7 & $1.80637 \mathrm{E}-05$ & 0 \\
\hline
\end{tabular}

En las tablas 3 y 4 se puede observar que la placa sin inhibidor presenta la mayor velocidad de corrosión en comparación con aquellas que tenían inhibidores aplicados. Estas placas presentaron la mayor cantidad de corrosión, debido al proceso de corrosión acelerado, como se observa en la figura 12.

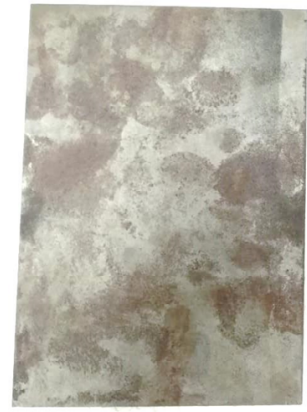

[a]

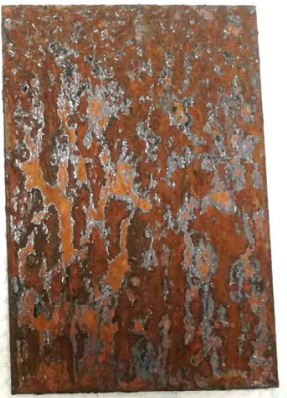

[b]
Figura 12. Comparación de placa sin exponer [a] y placa expuesta en la cámara de salinidad [b].

Para apreciar mejor los resultados de la pérdida de peso en las láminas de acero A36, se presentaron los pesos obtenidos al inicio y al final del proceso de decapado de las muestras. Como se presentan en la figura 13 que podemos ver a continuación.

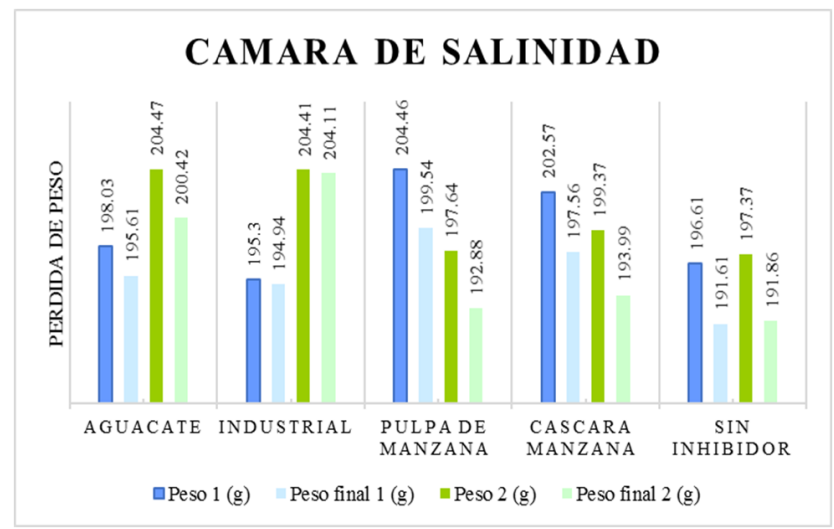

Figura 13. Cambios de peso en las láminas expuestas en la cámara de salinidad.

\section{Discusión}

La investigación cumple con el propósito de manera satisfactoria, ya que los inhibidores verdes procedentes de frutas fueron eficientes en los ambientes sometidos (costero natural y controlado). Afirmando así, que presentan tendencias anticorrosivas que ayudan a proteger los metales.

Se rechaza la hipótesis de investigación y se acepta la hipótesis nula porque las láminas con inhibidores verdes aplicados, de aguacate y manzana verde, muestran menor efectividad que los industriales. Sin embargo, es necesario considerar el menor impacto al ambiente de los inhibidores verdes, a base de frutas, y el potencial de inhibición de la corrosión que con mayor investigación y procesamiento de estos pueden mejorar su eficiencia.

Las láminas presentes en la cámara de salinidad estuvieron expuestas menor tiempo que las láminas presentes en la 
estación rompeolas, pero precisamente en ellas es donde se observa mayor corrosión.

El inhibidor verde, a base del extracto de aguacate, muestra mayor eficiencia en los dos sitios expuestos; mostrando una eficiencia en la cámara de salinidad de $52.21 \%$ a las 12 horas y $29 \%$ a las 24 horas. Mientras que en la estación rompeolas de Sherman muestra eficiencia de $41.46 \%$ en 144 horas y $66.37 \%$ en 408 horas.

\section{Conclusiones}

Acorde a los resultados obtenidos, podemos concluir:

En ambos sitios se muestra la siguiente tendencia de eficiencia de inhibidores con respecto al control:

- anticorrosivo industrial presentó mayor eficiencia debido a que es un producto debidamente estudiado y procesado.

- pulpa de aguacate fue el segundo con mayor eficiencia y el inhibidor verde más efectivo por la concentración de taninos que posee.

- pulpa de manzana en comparación con los dos anteriores no fue tan eficiente, pero si mostro ciertas tendencias anticorrosivas.

- cáscara de manzana este inhibidor verde presenta la menor eficiencia en casi todos los periodos de exposición, pero de igual forma puede ser usado para mitigar la corrosión.

Lo cual muestra que al tener este tipo de acero A36 expuesto cerca de ambientes marinos se deben utilizar recubrimientos anticorrosivos que les brinden mayor durabilidad.

Para próximas investigaciones se recomienda mayor tiempo de exposición de las láminas de acero A36, que nos ayuden a obtener mejores resultados aumentando la concentración de los extractos.

\section{AGRADECIMIENTO}

Agradecemos al Dr. Cecilio Hernández principal colaborador durante todo este proceso, quien con su conocimiento, enseñanza y disposición permitió el desarrollo de este proyecto.

Al Centro Experimental de Ingeniería de la Universidad Tecnológica de Panamá, por permitirnos utilizar los laboratorios. En especial el laboratorio de Análisis Industriales y Ciencias Ambientales (LABAICA), quien nos proporcionó los implementos necesarios para llevar a cabo la investigación.

\section{REFERENCIAS}

[1] Cecilio Hernández B., Estudio comparativo de los productos de la corrosión atmosférica del acero al carbono, tratados con extractos acuosos de productos naturales. [Mg.Tesis]. 2007, Universidad Nacional de Panamá.
[2] Latifa Hamadi, Salah Mansouri, Kafia Oulmi, Abdelhak Kareche. El uso de aminoácidos como inhibidores de la corrosión para metales: una revisión. Diario egipcio del petróleo. Volumen 27, Núm. 4, 2018, páginas 1157-1165

[3] Aprael S., Yaro Anees A., Khadom Rafal K. Wael. Jugo de albaricoque como inhibidor de la corrosión verde del acero suave en ácido fosfórico. Revista Alexandria Engineering. Volumen 52, Núm. 1, 2013, páginas 129-135.

[4] Paola García, Lizneth Guerra, Johana Quintero, Didiana Rodríguez, Yatzuri Sosa. Extracción del aceite de la cáscara de la nuez de la India (Anacardium Occidentale) para el tratamiento superficial de metales. Revista de Iniciación Científica. Vol.2 Núm. 2. 2016.

[5] Virginia Lidia Gomez Chacón. Eficiencia de un inhibidor verde extraído de cáscara de manzana mediante el sistema Soxhlet en la corrosión del acero 1018 en medio ácido. Tesis. 2016.

[6] María J. Berrocal B., Edgar D. Altamiranda P., Evaluación de la eficiencia inhibidora de corrosión del extracto acuoso de aguacate (Persea americana). [Tesis], 2013.

[7] Universidad Tecnológica de Panamá, Centro Experimental de Ingeniería. Uso de los sitios de exposición atmosférica ubicados en el antiguo fuerte Sherman, provincia de Colón.

[8] Juan Carlos Montes Atuesta. Caracterización ante la corrosión atmosférica del acero autoprotector A588. [Ing. Tesis], 2009. Universidad de los Andes, Bogotá, D.C.

[9] ASTM G-1, Preparing, Cleaning and Evaluating corrosion test Specimens, (International Standards Organization, Ginebra, 1992).

[10] ASTM B - 117, Standard Methods of Spay (FOG) testing, (International Standards Organization, Ginebra, 1986).

[11] ASTM G-31, Standard Practice for Laboratory Immersion Corrosion Testing of Metals, (International Standards Organization, Ginebra, 1986).

[12] Corrosividad atmosférica de Panamá. Proyecto MICATPanamá. Universidad tecnológica de Panamá, Universidad de Panamá. 